\title{
Long-Term Depression in the Developing Hippocampus: Low Induction Threshold and Synapse Nonspecificity
}

\author{
Pontus Wasling, Eric Hanse, and Bengt Gustafsson \\ Institute of Physiology and Pharmacology, Göteborg University, SE-405 30 Göteborg, Sweden
}

It was observed that the use of paired-pulse afferent stimulation as test stimulation $(0.1-0.02 \mathrm{~Hz})$ in the hippocampal CA1 area in young (1-2 week) rats, but not in older ones, led to declining synaptic activity. We show that such very low-frequency stimulation leads to long-term depression (LTD) initiated by activation of NMDA receptor channels and/or T-type voltagedependent calcium channels. The depression is initiated within three or four such stimuli, and $<10$ are sufficient to induce a notable long-term effect. When the paired-pulse stimulation exceeded threshold for postsynaptic spike activation, the depression was preceded by an NMDA receptor-dependent po- tentiation. Irrespective of whether homosynaptic potentiation or depression occurred, the paired pulse stimulation also induced depression in neighboring synapses alternately activated by single stimuli. These results point to a very high sensitivity for induction of synaptic depression during the neonatal period. They also support the notion that a brief rise in postsynaptic calcium can induce long-term potentiation (LTP) or LTD, a larger rise more likely to induce LTP, as well as that a prolonged modest increase produces selectively only LTD.

Key words: long-term depression; hippocampus; CA1; heterosynaptic; synaptic plasticity; development
After activation, synapses alter their efficacy, even after a single stimulus. Such paired-pulse (PP) plasticity is generally attributed to an alteration in presynaptic release probability after the first stimulus (Katz and Miledi, 1968; Zucker, 1989), lasts for $<1 \mathrm{sec}$, and is of consequence for the short-term computational properties of the synapse (Dobrunz and Stevens, 1999). PP stimulation is also often used as an experimental tool because the magnitude and direction of PP plasticity are sensitive to the state of release probability of the synapse (Manabe et al., 1993). However, PP stimulation has also been found to lead to more long-term synaptic plasticity effects. In disinhibited slices, even a single such stimulation, if strong enough, can lead to a significant long-term potentiation (Wigstrom and Gustafsson, 1985). Moreover, PP stimulation can facilitate the ability of a $1 \mathrm{~Hz}$ afferent activation to induce long-term depression (LTD) (Thiels et al., 1994).

In experiments using slices from young (1-2 week) rats we observed that the use of PP stimulation at low rates $(0.1 \mathrm{~Hz})$ as test stimulation led to difficulties in establishing a stable baseline. Thus, PP stimulation producing field EPSPs that were subthreshold for spike activation led to a decline in field EPSP magnitude. In the present report we demonstrate that this decline is caused by the development of LTD by such stimulation, not only of the PP-stimulated synapses, but also of neighboring synapses alternately activated by single stimuli.

\section{MATERIALS AND METHODS}

Slice preparation and solutions. Experiments were performed on hippocampal slices from 6- to 42-d-old rats $(n=121)$. The animals were anesthetized with isoflurane, decapitated, and their brains were removed to an ice-cold artificial CSF (ACSF) solution containing $6 \mathrm{~mm} \mathrm{Mg}^{2+}$ and

\footnotetext{
Received Aug. 13, 2001; revised Nov. 27, 2001; accepted Dec. 18, 2001.

This project was supported by the Swedish Medical Research Council (Project Numbers 05180 and 12600).

Correspondence should be addressed to Pontus Wasling, Institute of Physiology and Pharmacology, Göteborg University, Box 432, SE-405 30 Göteborg, Sweden. E-mail: pontus.wasling@physiol.gu.se.

Copyright (ㄷ) 2002 Society for Neuroscience $\quad 0270-6474 / 02 / 221823-08 \$ 15.00 / 0$
}

$0.5 \mathrm{mM} \mathrm{Ca}^{2+}$. Slices $(400-\mu \mathrm{m}$-thick) were obtained with a vibratome (Campden Instruments, Loughborough, UK) and stored in ACSF at room temperature. After at least $30 \mathrm{~min}$ of storage, a single slice was transferred to a recording chamber where it was kept submerged in a constant flow $(\sim 2 \mathrm{ml} / \mathrm{min})$ at $30-32^{\circ} \mathrm{C}$. The perfusion fluid contained (in $\mathrm{mm}$ ): $\mathrm{NaCl} 124, \mathrm{KCl} 3, \mathrm{CaCl}_{2} 4, \mathrm{MgCl}_{2} 4, \mathrm{NaHCO}_{3} 26, \mathrm{NaH}_{2} \mathrm{PO}_{4} 1.25$, and D-glucose 10 and was continuously bubbled with $95 \% \mathrm{O}_{2}$ and $5 \%$ $\mathrm{CO}_{2}, \mathrm{pH} \sim 7.4$. Bicuculline methiodide $(10 \mu \mathrm{M})$ was always present, unless otherwise stated, to block $\mathrm{GABA}_{\mathrm{A}}$ receptor-mediated activity. The higher than normal calcium and magnesium concentrations were used, and a surgical cut between CA1 and CA3 regions were made, to counteract the increased excitability in the presence of bicuculline. In some of the experiments in which bicuculline was not present, the higher concentrations of calcium and magnesium were still used for direct comparison of experiments with and without $\mathrm{GABA}_{\mathrm{A}}$ ergic inhibition. In other such experiments, calcium and magnesium were present at more physiological concentrations $(2 \mathrm{mM})$.

Recording and analysis. Electrical stimulation of afferent fibers and recordings of extracellular synaptic potentials were performed in the CA1 hippocampal region. Stimuli consisted of $0.2 \mathrm{msec}$ negative constant current pulses $(15-50 \mu \mathrm{A})$ delivered through bipolar tungsten wires. Stimulation electrodes were positioned in the stratum radiatum on either side of the recording electrode to provide two independent afferent inputs projecting to the same dendritic region. Both inputs received a test stimulus every 10 $\mathrm{sec}$, but $5 \mathrm{sec}$ apart. The stimulation intensity was set not to evoke firing in the postsynaptic neurons, unless otherwise stated, as evidenced by the absence of a population spike distorting the field EPSP.

Recordings were made by means of a glass micropipette (filled with 3 $\mathrm{M} \mathrm{NaCl}$ ) in stratum radiatum. Field EPSPs were amplified with an Axoclamp-2A (Axon Instruments, Foster City, CA) and filtered at $3 \mathrm{kHz}$. Data were digitized (10 kHz sampling rate) and collected using a 486 personal computer. Evoked responses were analyzed off-line using custom-made IGOR Pro (WaveMetrics, Lake Oswego, OR) software, written by Pontus Wasling. Field EPSP magnitude was estimated by linear regression over the first $0.8 \mathrm{msec}$ of the initial slope. The presynaptic volley was measured as the peak-to-peak amplitude of the initial positive-negative deflection, and it was not allowed to change by $>3 \%$ over the 40-60 min stimulation period. Statistical significance for paired and independent samples was evaluated using Student's $t$ test $(p<0.05)$.

Drugs used were: D-2-amino-5-phosphonopentanoate (D-AP-5) and $(R S)$-1-aminoindan-1,5-dicarboxylic acid (AIDA) from Tocris Neuramin (Bristol, UK), and bicuculline methiodide and $\mathrm{NiCl}_{2}$ from Sigma-Aldrich (Stockholm, Sweden). 


\section{RESULTS}

When in a 4- to 5-week-old rat afferent stimulation at $0.1 \mathrm{~Hz}$ shifted from a single to a PP stimulus (50 msec interval) for 20 min, field EPSP slopes remained much the same during as well as after the PP stimulus epoch (Fig. 1A) $(104 \pm 3 \% ; n=6 ; 20 \mathrm{~min}$ after end of PP stimulation). However, when performing the same experiment in younger rats (6-12 d), field EPSPs started to depress during the PP stimulation and remained thereafter depressed (Fig. 1B). This depression could be followed for the duration of the recording, in some cases up to $2 \mathrm{hr}$, and is thus an LTD. It was not associated with any change in the afferent volley (see Materials and Methods) and is thus an LTD of synaptic transmission. In 6- to 12-d-old rats the field EPSPs 20 min after cessation of PP stimulation had decreased to $71 \pm 2 \%$ of baseline $(n=8)$. Also when examined in somewhat older rats, $17-18 \mathrm{~d}$, LTD, albeit smaller, could be evoked $(77 \pm 3 \% ; n=9)$. After two 20 min PP stimulation epochs (20 min interval) in 6- to 12-d-old rats field EPSPs were further depressed (to $48 \pm 3 \% ; n=3$ ). However, additional stimulation epochs did not produce more depression, indicating that the LTD was saturable.

When examined in the younger rats, LTD occurred using a range of test frequencies and $\mathrm{PP}$ intervals. Thus, stimulation at $0.033 \mathrm{~Hz}$ led to a LTD similar in development (per PP stimulus) to that at $0.1 \mathrm{~Hz}$ (Fig. 1C), and even a $0.016 \mathrm{~Hz}$ test frequency led to an LTD (data not shown). Similarly, when using longer PP intervals (200-1000 msec) LTD, albeit smaller $(81 \pm 3 \% ; n=6)$, was observed. Similar values were also found at PP intervals of 2.5 $\sec (80 \pm 4 \% ; n=7)$.

The above experiments were performed in the presence of the $\mathrm{GABA}_{\mathrm{A}}$ receptor antagonist bicuculline to allow for the analysis of changes in the excitatory transmission alone. The presence of disinhibition was, however, not a prerequisite to observe PPinduced LTD. In fact, in the absence of bicuculline, PP stimulation led to an even larger LTD $(57 \pm 5 \% ; n=5)$. Neither was the use of higher than normal calcium and magnesium concentrations (4 mM; see Materials and Methods) necessary for the observation of this LTD. Thus, in the absence of bicuculline and in $2 \mathrm{~mm}$ calcium and magnesium in the perfusion solution, the LTD was of about similar magnitude (71 $\pm 3 \% ; n=5)$.

\section{Induction of PP LTD involves both NMDA receptors and T-type calcium channels}

To examine the pharmacology of the induction of this LTD, 8- to 13-d-old rats were examined, the PP interval was kept at $50 \mathrm{msec}$, and bicuculline was used to block $\mathrm{GABA}_{\mathrm{A}}$ receptor-mediated inhibition, unless otherwise stated. In the presence of the NMDA receptor antagonist D-AP-5 (50-100 $\mu \mathrm{M})$, PP stimulation led to an LTD that was smaller, although not significantly so $(78 \pm 2 \%, n=$ $10 ; p>0.05$ ) (Fig. 2A). T-type voltage-gated calcium channels can be involved in the induction of LTD, in particular a form observed when synaptic inhibition is not blocked (Oliet et al., 1997). Application of the T-type calcium channel antagonist $\mathrm{Ni}^{2+}$ $(100 \mu \mathrm{M})$ to the disinhibited slice, like D-AP-5, led to a small, and nonsignificant, decrease of LTD $(78 \pm 2 \%, n=7 ; p>0.05)$ (Fig. $2 B)$. The L-type voltage-gated calcium channel antagonist nifedipine $(30-50 \mu \mathrm{M})$ did not reduce the LTD $(69 \pm 8 \% ; n=4)$.

In the absence of bicuculline, both $\mathrm{D}-\mathrm{AP}-5$ and $\mathrm{Ni}^{2+}$ significantly reduced the LTD, from the $57 \%( \pm 5 ; n=5)$ observed in control solution to $87 \pm 3 \%(n=8$; D-AP-5) and $75 \pm 3 \%(n=$ 5 ; $\left.\mathrm{Ni}^{2+}\right)$. This larger reduction of LTD by AP- 5 and $\mathrm{Ni}^{2+}$ than in the disinhibited slice may be a consequence of the fact that NMDA receptor channels and voltage-gated calcium channels
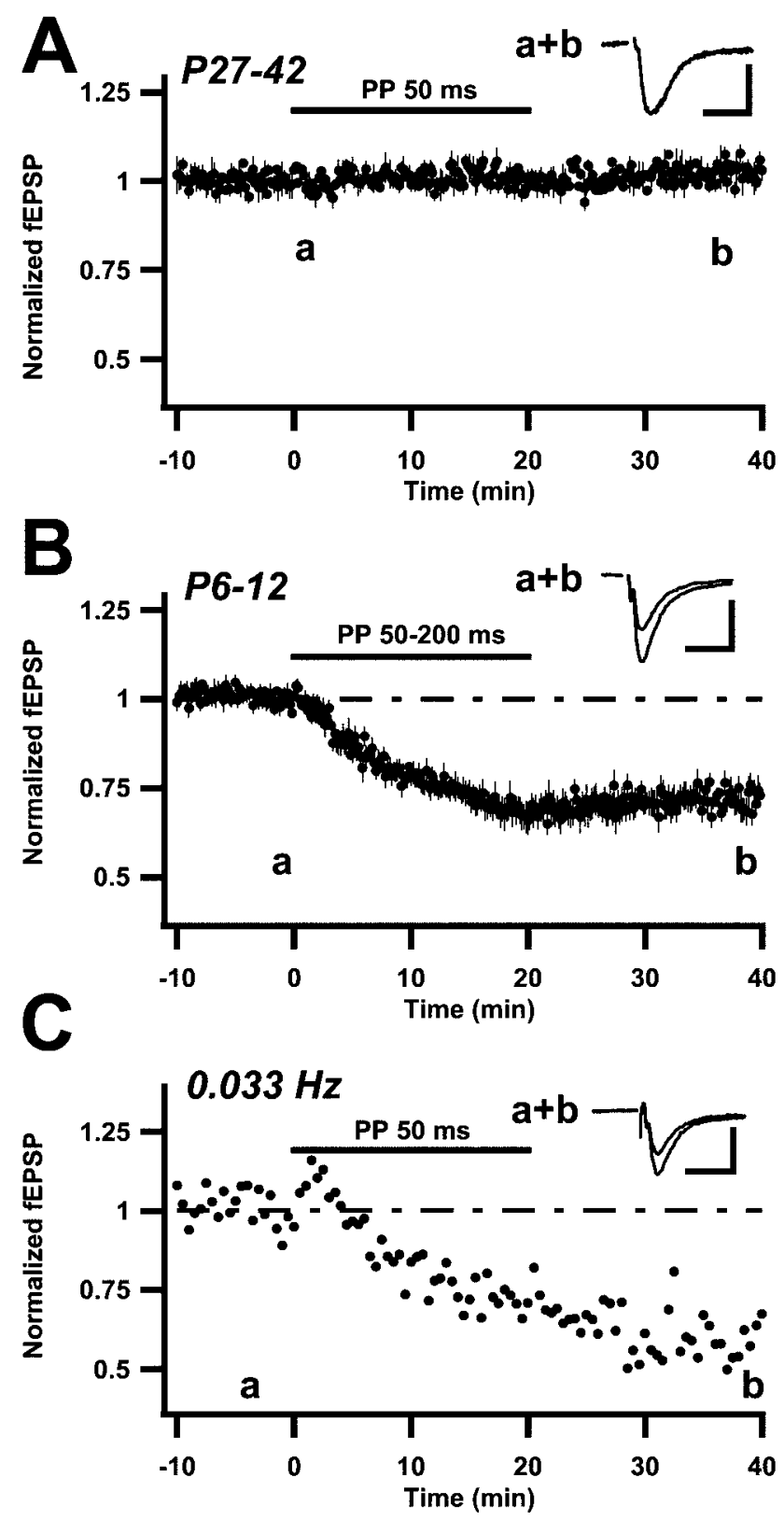

Figure 1. PP stimulation elicits LTD in young but not in older rats. $A-C$, Summary plots of normalized initial slope measurements of field EPSPs, evoked in the stratum radiatum layer of the hippocampal CA1 region. Stimulation rate is $0.1(A, B)$ and $0.033 \mathrm{~Hz}(C)$. The horizontal bars indicate the time period during which single volley test stimulation was replaced by PP stimulation. The slope measurements are normalized with respect to the 2 min period immediately preceding the onset of PP stimulation. In $A$ and $B$ average values \pm SEM are shown. Insets are the averages of 12 sweeps taken from representative experiments, at a time indicated by the characters in the graph. Calibration: $0.2 \mathrm{mV}, 10 \mathrm{msec} . A$, PP stimulation is applied for $20 \mathrm{~min}$ at $0.1 \mathrm{~Hz}$ in 27 - to 42 -d-old rats $(n=$ 6). $B$, PP stimulation is applied for $20 \mathrm{~min}$ at $0.1 \mathrm{~Hz}$ in $6-12 \mathrm{~d}$ old rats. Data are pooled from experiments using PP interstimulus intervals of 50 $\operatorname{msec}(n=4), 100 \mathrm{msec}(n=2)$, and $200 \mathrm{msec}(n=2)$. $C$, From one experiment, using a 10-d-old rat, showing the effect of PP stimulation when the test frequency was $0.033 \mathrm{~Hz}$.

interact in a dual manner in that they both permeate calcium ions in a voltage-dependent manner and also produce depolarization. Blockade of one type of channel would then be expected to cause a supralinear reduction of the calcium signal because of less 

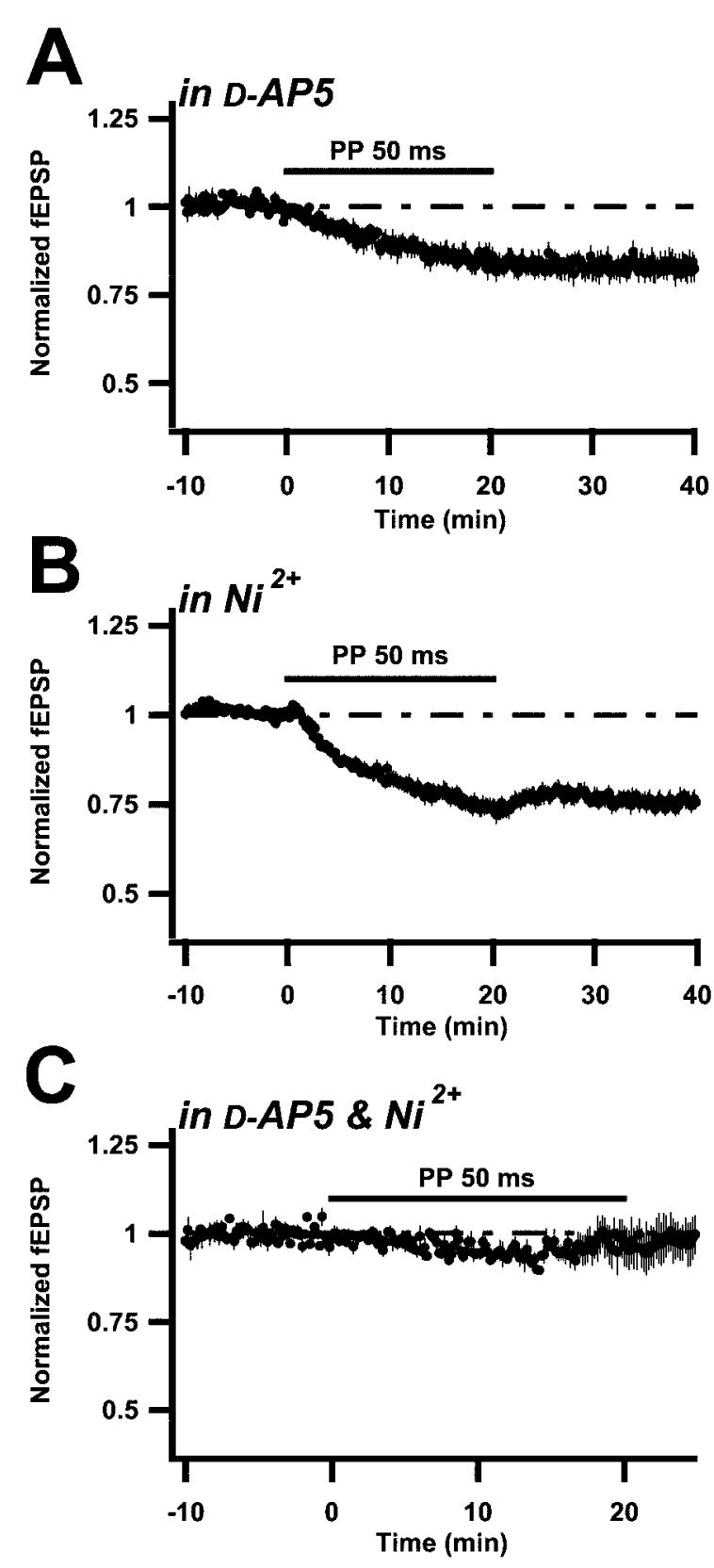

Figure 2. Induction of LTD elicited by PP stimulation involves both NMDA receptor channels and T-type $\mathrm{Ca}^{2+}$ channels. $A-C$, Summary plots as described in Results and in Figure 1. All experiments were performed on 8- to 13 -d-old rats. $A$, PP stimulation was applied in the presence of $(50-100 \mu \mathrm{M})$ D-AP-5 $(n=10) . B$, PP stimulation was applied in the presence of $100 \mu \mathrm{M} \mathrm{Ni}^{2+}(n=7)$. $C$, PP stimulation was applied in the presence of both D-AP-5 $(50 \mu \mathrm{M})$ and $\mathrm{Ni}^{2+}(100 \mu \mathrm{M})(n=5)$.

overall depolarization. It is not unlikely that such an effect is larger when inhibition is present, making the overall depolarization during synaptic activation less (Hanse and Konnerth, 1998).

Because both $\mathrm{D}-\mathrm{AP}-5$ and $\mathrm{Ni}^{2+}$ seemed able to reduce the amount of LTD produced, it was examined whether the coapplication of these antagonists could more fully block LTD. In fact, in such experiments (in the presence of bicuculline) LTD was reduced to an almost nonsignificant level (94 $\pm 2 \% ; n=5$ ) (Fig. $2 C$ ). On the other hand, coapplication of D-AP-5 and nifedipine led to an LTD $(84 \pm 4 \% ; n=6)$ not significantly smaller than that found in D-AP-5 alone. Metabotropic glutamate receptors
(mGlurs) have been implicated in some forms of LTD (Bolshakov and Siegelbaum, 1994; Bortolotto et al., 1994; Kemp and Bashir, 1997; Oliet et al., 1997; Kemp and Bashir, 1999). However, after coapplication of the mGlur antagonist AIDA $(500 \mu \mathrm{M})$ and D-AP-5 $(50 \mu \mathrm{M})$, LTD was not smaller than in the presence of D-AP-5 alone $(78 \pm 3 \% ; n=3)$.

The reduced LTD observed in the presence of D-AP-5 and $\mathrm{Ni}^{2+}$ was not secondary to reductions of field EPSP amplitude resulting from application of these drugs. In these experiments the stimulation strength was set to evoke similarly sized baseline field EPSPs, and the 10-20\% reduction of the field EPSP by $\mathrm{Ni}^{2+}$ (none by D-AP-5) was thus compensated for. On average, the field EPSP peak amplitudes in control solution, in D-AP-5, in $\mathrm{Ni}^{2+}$, and in D-AP-5 combined with $\mathrm{Ni}^{2+}$ were $0.39,0.34,0.37$, and 0.35 $\mathrm{mV}$, respectively.

\section{LTD can be preceded by an initial potentiation}

When PP stimulation was strong enough to produce postsynaptic spike activity (Fig. $3 A$, inset), the LTD was preceded by a potentiation of the field EPSP slope (Fig. $3 A$ ). However, the final level of depression (20 min after end of PP stimulation) was much the same $(75 \pm 1 \% ; n=9)$ as when this potentiation did not occur. In Figure $3 B$ are illustrated results from experiments in which only a brief episode (nine stimuli) of PP stimulation was given. In experiments in which subthreshold stimuli were used (inset b) depression resulted, whereas those in which spikes were evoked (inset $a$ ) potentiation was observed. In the presence of D-AP-5 (50 $\mu \mathrm{M})$, such strong PP stimulation (insert) did not result in any initial potentiation, only in LTD (Fig. $3 C$ ). On the other hand, $\mathrm{Ni}^{2+}(100 \mu \mathrm{M})$ did not block this initial potentiation (Fig. 3D). PP stimulation (nine PP stimuli) thus seems capable of initiating not only LTD but also NMDA receptor-dependent potentiation. It can be noted that although being of much smaller initial magnitude, the LTD evoked by this brief PP stimulation was much more stable than the corresponding potentiation.

LTD, but not initial potentiation, is synapse-nonspecific In the present experiments, stimulation was alternated between two electrodes positioned in the dendritic layer on either side of the recording electrode, the stimuli being $5 \mathrm{sec}$ apart. When PP stimulation was initiated at one of these stimulation sites, the field EPSP, but not the afferent volley, produced by stimulation at the other site also started to become depressed (Fig. 4A). Twenty minutes after the cessation of PP stimulation, the control input was still depressed to $88 \pm 4 \%(n=8)$ of baseline value, i.e., demonstrating an LTD of approximately one-third of that of the conditioned input. When stimulation was such that an initial potentiation occurred, this potentiation was not seen in the control input (Fig. 4B). Thus, depression of the control input is not secondary to overlap between the fiber populations activated by the two stimulation electrodes. That is, the LTD, in contrast to the potentiation, is not specific for the PP-activated synapses.

This heterosynaptic LTD was observed in the presence of D-AP-5 (50 $\mu \mathrm{M})$ (Fig. $4 C$ ) as well as in the presence of $\mathrm{Ni}^{2+}(100$ $\mu \mathrm{M})$ (Fig. 4D). Thus, activation of both NMDA receptor channels and T-type calcium channels can support the spread of LTD to unconditioned synapses. After coapplication of D-AP-5 and $\mathrm{Ni}^{2+}$, no LTD was observed (98 $\pm 4 \% ; n=4)$.

\section{Onset of LTD}

As can be noted in Figures 1-4, field EPSPs started to depress within the first minute of PP stimulation. Because PP stimulation per se did not produce field EPSP changes (Fig. 2C), it would 

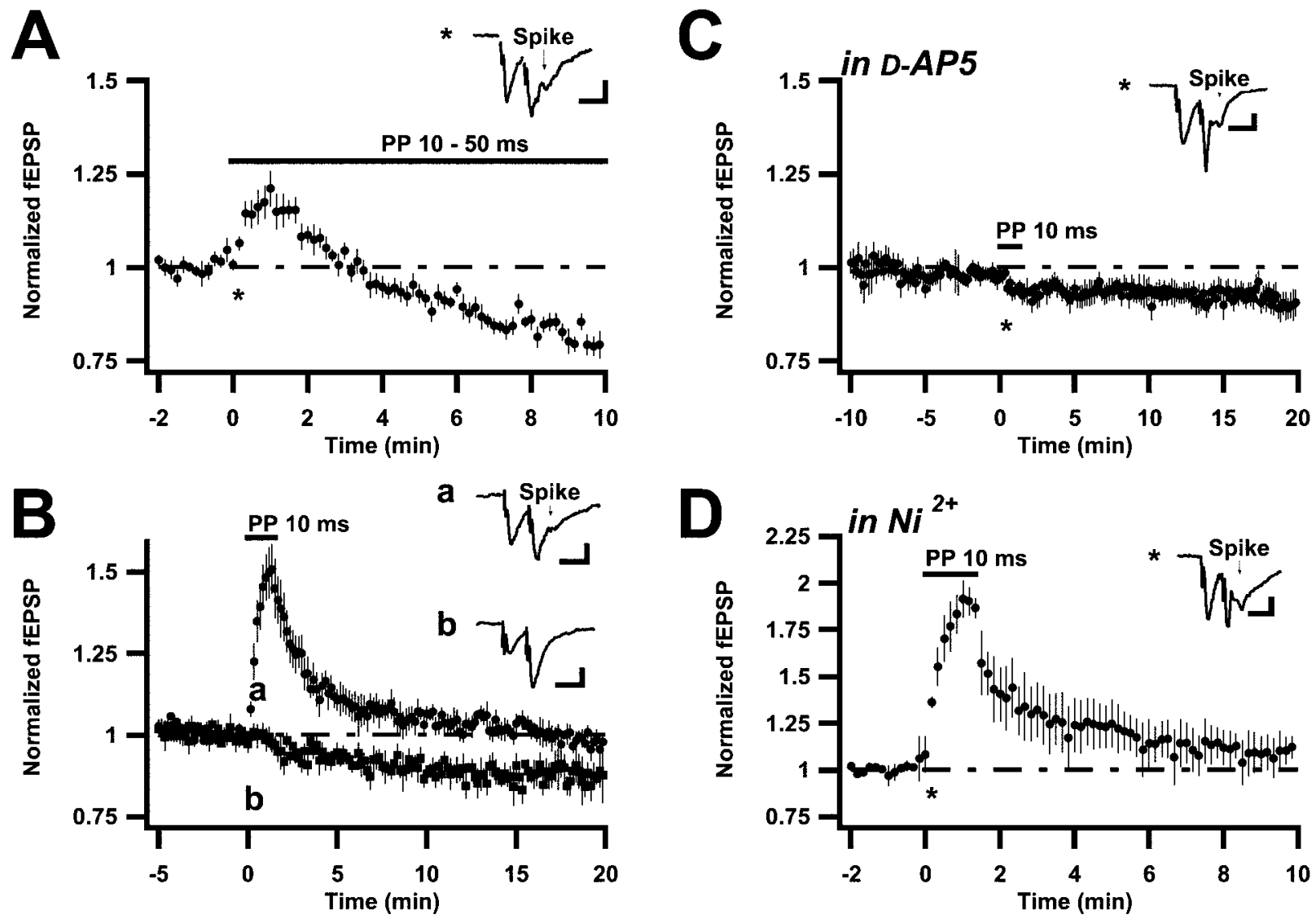

Figure 3. PP stimulation producing population spike activity elicits an initial NMDA receptor-dependent potentiation preceding the depression. $A-D$, Summary plots as described in Results and in Figure 1. Insets are averages of three sweeps taken from representative experiments, at the time indicated by the characters. Calibration: $0.2 \mathrm{mV}, 10 \mathrm{msec} . A$, PP stimulation (interstimulus interval $10-50 \mathrm{msec}$ ) associated with evident population spikes leading to an initial potentiation $(n=9)$. B, Data from experiments in which PP stimulation did $(a ; n=5)$, and did not $(b ; n=5)$ give rise to population spike activity are shown superimposed. Only nine consecutive PP stimulations (interstimulus interval $10 \mathrm{msec}$ ) were given. $C$, Nine consecutive PP stimulations (interstimulus interval $10 \mathrm{msec}$ ) associated with population spike activity were given in the presence of $50 \mu \mathrm{M}$ D-AP-5 $(n=7)$. Note the absence of an initial potentiation. $D$, The same but in the presence of $100 \mu \mathrm{M} \mathrm{Ni}{ }^{2+}(n=2)$. Note the large initial potentiation.

appear that LTD is rapidly induced, as also indicated by the stability of the depression after interruption of PP stimulation after a few stimuli (Fig. 3B,C). In Figure 5 are shown, on an expanded time scale, LTDs evoked in the test input at subthreshold PP stimulations $(A)$, in the control input $(B)$, and in the test input in the presence of D-AP-5 to ascertain the absence of an initial potentiation component $(C)$. On balance, these curves would suggest an onset of LTD within three or four stimuli, i.e., within $\sim 30$ sec.

\section{PP-induced LTD is associated with changes in PP facilitation}

The use of PP stimulation to induce LTD also allowed for the estimation of possible changes in PP plasticity during the development of LTD. Because there was no baseline PP stimulation, changes occurring in PP facilitation (PPF) during the development of LTD were normalized with respect to the average PPF during the first 10 PP stimulations. As can be noted in Figure 6, the development of LTD is paralleled by an increase in PPF that reached $16 \pm 3 \%(n=6)$ at the end of PP stimulation. A reduction of the EPSP by a decrease in stimulation strength (to $64 \%$ of control i.e., to approximately the same as an LTD) did not alter the PP ratio ( $101 \%$ of control; $n=7)$. The increase in PPF in association with LTD was thus not merely a consequence of the reduction in field EPSP magnitude. An increase of PPF was observed both for the LTD observed in the presence of D-AP-5 $(9 \pm 3 \% ; n=7)$ as well as of $\mathrm{Ni}^{2+}(19 \pm 6 \% ; n=8)$. In a few experiments PP stimulation was repeated after $\sim 20$ min, demonstrating that the increase in PPF remained throughout this period.

\section{DISCUSSION}

It has been a common observation that LTD is most easily generated in young rats (Dudek and Bear, 1993; Wagner and Alger, 1995), i.e., during a period when synapses are rapidly formed or eliminated (anatomically and/or functionally). LTD may then be part in such developmental synaptic regulation during this period. The present work demonstrates that in very young (1-2 weeks) rats, but not in older ones, a weak paired synaptic activation, even when infrequently occurring (0.1-0.02 $\mathrm{Hz}$ ), induces LTD. This weak induction stimulus was also found to be sufficient to induce LTD in synapses activated by single stimuli alternately with the PP-activated ones. These results point to a very high readiness to suppress very low-active synapses (and their neighbors) in the developing hippocampal synaptic network. As a result, low-activated cells in the developing hippocampal circuitry will be further deprived of their afferent activation.

Activation of both NMDA receptor channels and $\mathrm{Ni}^{2+}$. sensitive channels was found to initiate the induction of this LTD, 
A

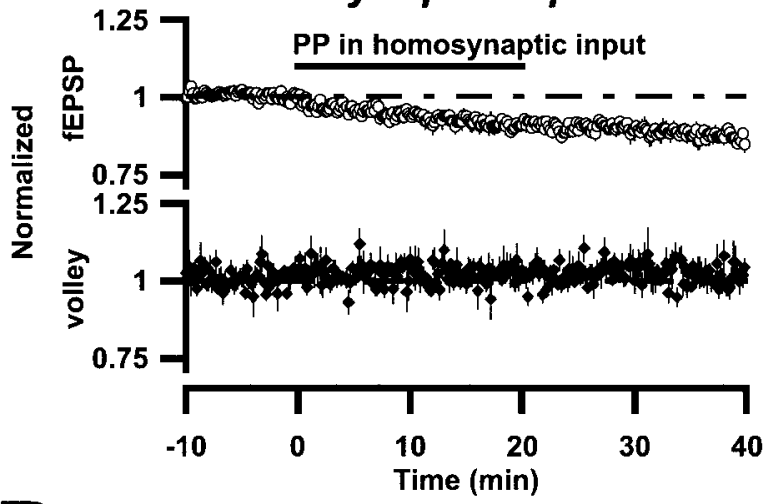

\section{Heterosynaptic input}

B

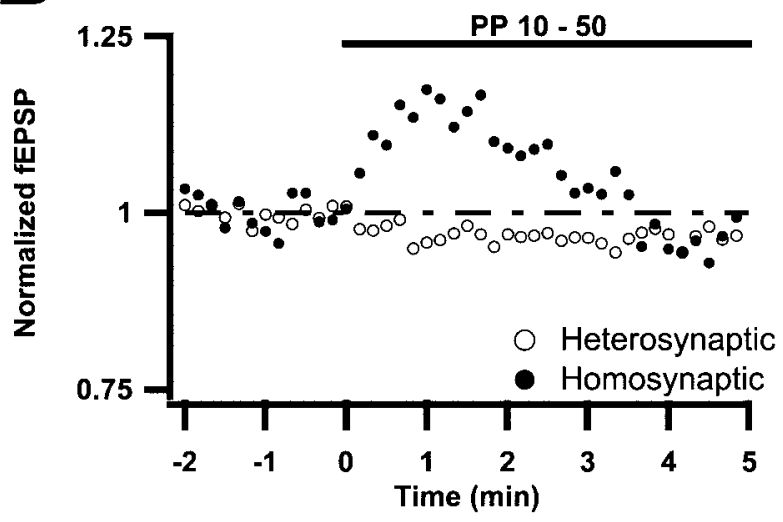

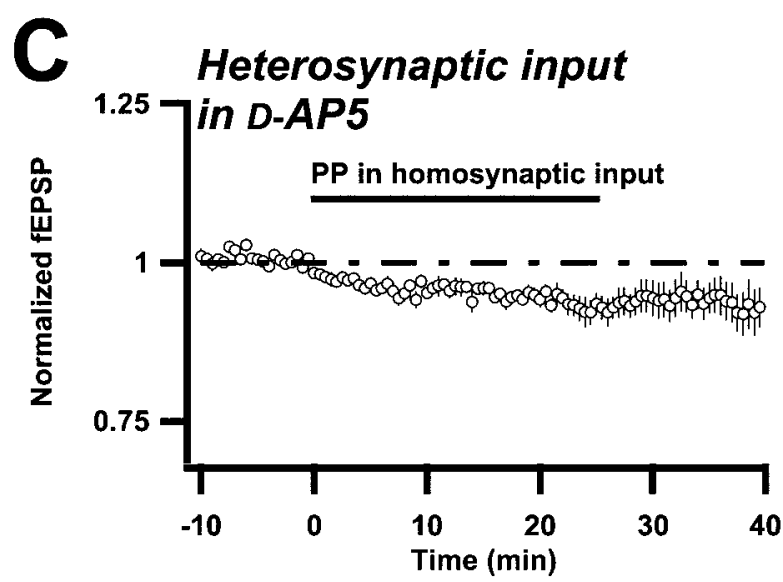

D

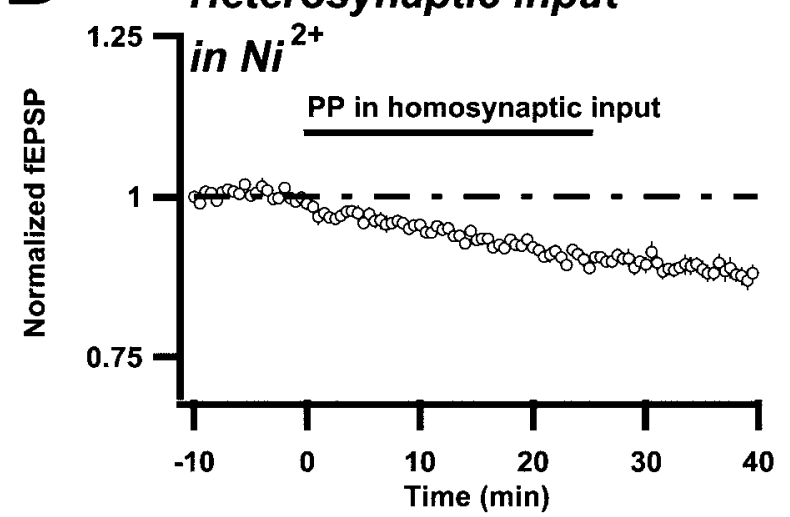

Figure 4. PP stimulation induces a synapse specific potentiation, but a synapse nonspecific depression. $A-D$, Summary plots as described in Results and in Figure 1. The two afferent inputs projecting to the same dendritic region were alternately stimulated at $0.1 \mathrm{~Hz}$. A, Top, Field EPSP changes occurring in one of the inputs (heterosynaptic), when the other input (homosynaptic) was subjected to PP stimulation $(n=8)$. Bottom, Same but for the presynaptic volley. $B$, Experiments in which PP stimulation was associated with population spike firing $(n=6)$. Note that the initial potentiation observed in the homosynaptic input (closed circles) is absent in the heterosynaptic one (open circles). $C$, Same as in $A$, but in the presence of $50 \mu \mathrm{M}$ D-AP-5. Note that each plotted value is the average of three consecutive field EPSPs $(n=7)$. $D$, Same as in $C$, but in the presence of $100 \mu \mathrm{M} \mathrm{Ni}{ }^{2+}(n=7)$. Note that the error bars are within the symbols.

the homosynaptic as well as the heterosynaptic one. Considering the small requirement for postsynaptic activity for the induction the $\mathrm{Ni}^{2+}$-sensitive channels are likely to be T-type voltage-gated calcium channels. This notion is supported by the observation that a greater amount of LTD was observed when postsynaptic inhibition was intact, a result likely explained by a larger possibility for deinactivation of such channels. Moreover, the involvement of low-voltage-activated calcium channels of L-type is less likely because nifedipine had little effect on this LTD.

The PP-induced LTD appears, with respect to its calcium sources for induction, not to resemble any previously described LTD. The limited effect of NMDA receptor antagonism (by itself), the dependence also on T-type calcium channels, and the heterosynaptic nature, sets the PP-induced LTD apart from lowfrequency (1-2 Hz) induced LTD (Dudek and Bear, 1992; Mulkey and Malenka, 1992). The small effect of T-type channel blockade (by itself), the lack of effect of metabotropic glutamate receptor antagonism, and its presence in disinhibited slices distinguishes the PP-induced LTD from the LTDs described by Oliet et al. (1997), and Bolshakov and Siegelbaum (1994). Thiels et al. (1994) and Kemp and Bashir (1997) reported using a standard low-frequency protocol that PP stimulation was more efficient to induce LTD than were single stimuli. However, this LTD was observed also in older rats, was homosynaptic (see also Huber et al., 2000), and in the younger rats it was fully blocked by NMDA receptor antagonists.

\section{PP-induced LTD and potentiation: differential thresholds}

LTD is most commonly induced by prolonged (10 min) lowfrequency activation, leading to the notion that its induction depends on a small but prolonged increase of postsynaptic calcium. However, brief high-frequency tetanization during partial NMDA receptor blockade can also produce LTD (Cummings et al., 1996). Yang et al. (1999) have suggested, based on flash photolysis of calcium, that a brief rise (seconds) in postsynaptic calcium can induce either long-term potentiation (LTP) or LTD, a larger rise more likely to induce LTP. However, a prolonged modest increase produces selectively only LTD. The present study supplies three findings that appear of interest in this context. A first is that PP activation at intervals of $10-60 \mathrm{sec}$ can induce LTD. This would seem to imply that a single PP activation by itself must supply sufficient calcium to induce LTD because calcium will hardly accumulate intracellularly with successive activation at such intervals. In 


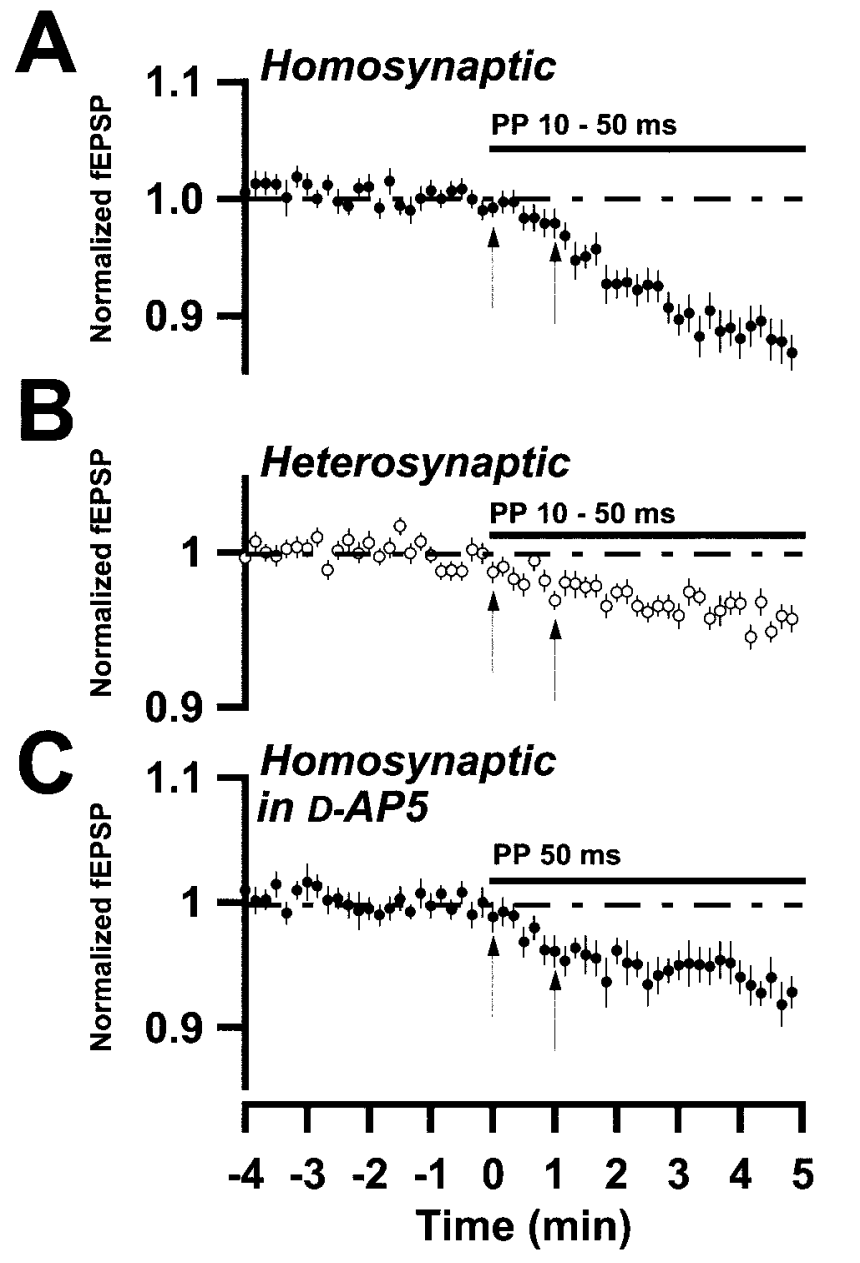

Figure 5. Onset of LTD induced by PP stimulation. $A-C$, Summary plots as described in Results and in Figure 1. $A$, Data from experiments in which PP stimulation produced no initial potentiation $(n=23)$. $B$, Same as in $A$, but for a heterosynaptic input $(n=26)$. $C$, Same as in $A$, but in the presence of D-AP-5 $(50-100 \mu \mathrm{M})(n=17)$. The vertical lines denote the time of PP stimulation onset, and 1 min thereafter, respectively. Note in $A-C$ that the depression is initiated already within $\sim 30 \mathrm{sec}$, i.e., within three or four stimuli.

other words, single instances of PP synaptic activation, subthreshold for postsynaptic spike generation, will have to suffice to activate NMDA receptor channels and/or T-type calcium channels to such an extent that the resultant calcium influx can set in motion (enzymatic) processes leading to LTD. Such sensitivity is also evidenced by the fact that the depression is already apparent within the first four PP stimuli.

A second finding is that stronger PP stimulation that led to postsynaptic spike activity elicited an NMDA receptordependent potentiation rather than LTD. As for the LTD, this potentiation became initiated within the very first few stimuli. This result is then in line with the above notion that "a brief rise in postsynaptic calcium can induce either LTP or LTD, a larger rise more likely to induce LTP." Interestingly, this potentiation was not reduced by $\mathrm{Ni}^{2+}$ and was fully blocked by an NMDA receptor antagonist. This result agrees with the idea that potentiation is more discriminative with respect to its calcium source than is depression (Hanse and Konnerth, 1998). Moreover, under these stimulus conditions, postsynaptic spikes appear to play a decisive role as to whether NMDA receptor
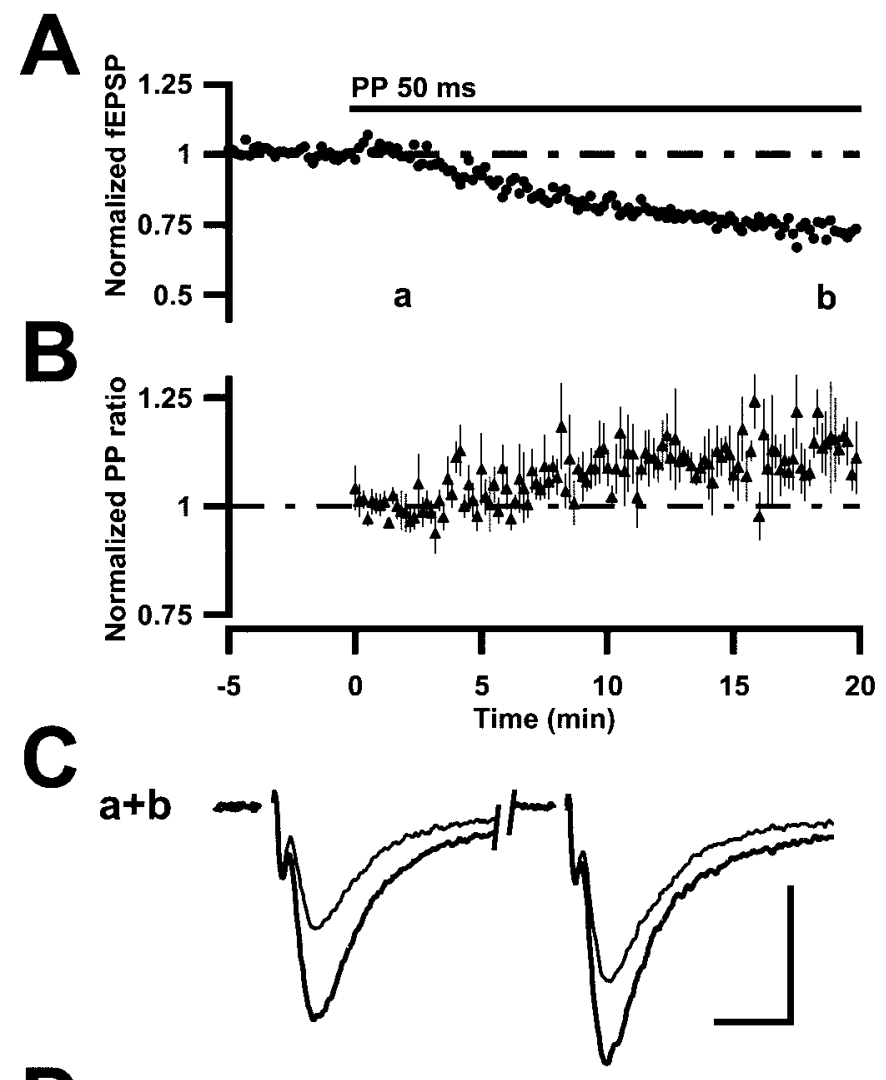

D

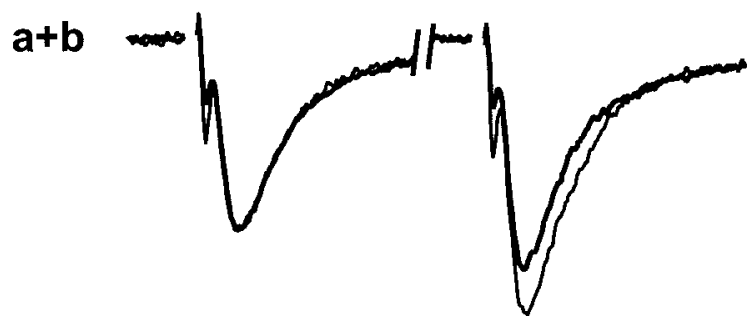

Figure 6. PP-induced LTD is associated with an increase in PPF. $A$, Summary plot, as described in Results and in Figure $1(n=6)$. B, PPF, normalized with respect to the average value obtained during the first 100 sec of PP stimulation, is plotted for the experiments illustrated in $A$. $C$, Averaged field EPSPs $(n=5)$ from a representative experiment taken at the times indicated by the characters ( $a$; thick line, $b$; thin line). Calibration: $0.2 \mathrm{mV}, 5 \mathrm{msec}$. $D$, Field EPSPs in $C$ are shown after normalization with respect to the first EPSP.

activation will be sufficient for induction of potentiation, or lead to depression.

Finally, if the strong PP stimulation was interrupted after a few stimuli, the potentiation slowly (10-20 min) decayed back toward baseline values, a decay that can be seen when tetanic stimulation is too brief or too weak to elicit LTP (Hanse and Gustafsson, 1994). This instability, which contrasts to the stable depression elicited by a similar number of stimuli subthreshold for spike activation, points to a greater intrinsic stability of the processes underlying LTD than LTP. Moreover, in agreement with the above notion "a prolonged modest increase produces selectively only LTD," continuation of the strong PP stimulation led to the replacement of the potentiation, within $5 \mathrm{~min}$, of a LTD. In other words, a stimulus event strong enough to induce potentiation did, by its repetition alone, initiate processes leading to LTD. 
Prolongation of a $5 \mathrm{~Hz}$ train can affect the LTP observed using a briefer train (Thomas et al., 1996). In that case, however, only depotentiation occurred. The transformation from potentiation to depression by prolonging a given (afferent) stimulus situation has to our knowledge not been described previously. However, using repeated flash photolysis of calcium, Yang et al. (1999) (their Fig. 2A) seemingly demonstrated such transformation. The present results do however only demonstrate that the transformation from potentiation to depression takes place among a population of synapses, not within a synapse. Stronger stimulation may induce potentiation in some synapses while others still become depressed, prolongation altering the balance between these two effects. Our results can thus not be taken as strong evidence for a threshold shift for LTD in an individual synapse by prolonged activation. However, it was noted that the amount of final LTD was the same, whether an initial potentiation occurred, or not, a result seemingly favoring the idea that the transformation takes place at the level of a single synapse. Otherwise, one would have to assume that the depressed synapses must be considerably more depressed than in the absence of potentiation.

\section{Input nonspecificity}

What seems also particular with this LTD is its lack of synapse specificity. LTDs previously reported, using stimulation subthreshold for postsynaptic spike generation, have been described as being input specific (Dudek and Bear, 1992; Mulkey and Malenka, 1992). However, after strong postsynaptic activation leading to LTP induction, LTD can be induced in a nonactivated input (Lynch et al., 1977; Abraham and Goddard, 1983; Scanziani et al., 1996). This behavior has been explained as spread of depolarization (Staubli and Ji, 1996), or of calcium (Teyler et al., 1995), within the dendrite, from activated spines to neighboring synapses, or as an extracellular diff usion of a substance produced in activated neurons (Scanziani et al., 1996). For the present weak stimulation, such explanations appear unlikely. However, recently, heterosynaptic LTD has also been described after weaker stimulation leading to homosynaptic LTD. This nonspecificity is explained on the basis of intradendritic spread of calcium mediated via $\mathrm{InsP}_{3}$-operated calcium stores (Nishiyama et al., 2000). Such an explanation may then also apply for the presently observed heterosynaptic LTD. However, the heterosynaptic LTD described by Nishiyama et al. (2000) required postsynaptic spike activity for its induction, and it was of the same magnitude as the homosynaptic one, not approximately one-third of it as presently found. Moreover, it was not observed in association with homosynaptic LTP. In the present study the heterosynaptic LTD was approximately the same regardless of whether homosynaptic depression or potentiation was produced. It seems thus premature to attribute the same mechanism for the presently observed heterosynaptic LTD as for that described by Nishiyama et al. (2000).

\section{Which is the implication of the associated changes in PPF?}

Hippocampal LTD is generally thought to have a postsynaptic expression based on a reduction in AMPA receptor number (Malenka and Nicoll, 1999). The PP-induced LTD was however associated with an increase of PPF, indicating a change in presynaptic release probability. Results pointing toward a presynaptic locus of LTD expression have been reported previously in hippocampal synapses (Bolshakov and Siegelbaum, 1994; Stevens and Wang, 1994). In corticostriatal synapses LTD has also been found to be associated with an increased PPF (Choi and Lovinger, 1997; Egger et al., 1999). While being supportive of a presynaptic expression, the presently observed PPF changes do not constitute decisive evidence for this notion. Because these hippocampal synapses vary considerably with respect to initial release probability and to PPF magnitude (Dobrunz and Stevens, 1997), any bias introduced by this variation with respect to LTD induction will introduce PPF changes. For example, if LTD is mostly generated in synapses exhibiting small PPF values, the PPF would on average increase in the population. Because we do not know the exact conditions for the induction of this LTD in a given synapse, the possibility of a selective induction cannot be excluded.

\section{REFERENCES}

Abraham WC, Goddard GV (1983) Asymmetric relationships between homosynaptic long-term potentiation and heterosynaptic long-term depression. Nature 305:717-719.

Bolshakov VY, Siegelbaum SA (1994) Postsynaptic induction and presynaptic expression of hippocampal long- term depression. Science 264:1148-1152.

Bortolotto ZA, Bashir ZI, Davies CH, Collingridge GL (1994) A molecular switch activated by metabotropic glutamate receptors regulates induction of long-term potentiation. Nature 368:740-743.

Choi S, Lovinger DM (1997) Decreased probability of neurotransmitter release underlies striatal long-term depression and postnatal development of corticostriatal synapses. Proc Natl Acad Sci USA 94:2665-2670.

Cummings JA, Mulkey RM, Nicoll RA, Malenka RC (1996) Ca2+ signaling requirements for long-term depression in the hippocampus. Neuron 16:825-833.

Dobrunz LE, Stevens CF (1997) Heterogeneity of release probability, facilitation, and depletion at central synapses. Neuron 18:995-1008.

Dobrunz LE, Stevens CF (1999) Response of hippocampal synapses to natural stimulation patterns. Neuron 22:157-166.

Dudek SM, Bear MF (1992) Homosynaptic long-term depression in area CA1 of hippocampus and effects of $N$-methyl-D-aspartate receptor blockade. Proc Natl Acad Sci USA 89:4363-4367.

Dudek SM, Bear MF (1993) Bidirectional long-term modification of synaptic effectiveness in the adult and immature hippocampus. J Neurosci 13:2910-2918.

Egger V, Feldmeyer D, Sakmann B (1999) Coincidence detection and changes of synaptic efficacy in spiny stellate neurons in rat barrel cortex. Nat Neurosci 2:1098-1105.

Hanse E, Gustafsson B (1994) Onset and stabilization of NMDA receptor-dependent hippocampal long-term potentiation. Neurosci Res 20:15-25.

Hanse E, Konnerth A (1998) Calcium and activity-dependent synaptic plasticity. In: Integrative aspects of calcium signalling (Verkhratsky A, Toescu EC, eds), pp 333-358. New York: Plenum.

Huber KM, Kayser MS, Bear MF (2000) Role for rapid dendritic protein synthesis in hippocampal mGluR- dependent long-term depression. Science 288:1254-1257.

Katz B, Miledi R (1968) The role of calcium in neuromuscular facilitation. J Physiol (Lond) 195:481-492.

Kemp N, Bashir ZI (1997) NMDA receptor-dependent and -independent long-term depression in the CA1 region of the adult rat hippocampus in vitro. Neuropharmacology 36:397-399.

Kemp N, Bashir ZI (1999) Induction of LTD in the adult hippocampus by the synaptic activation of AMPA/kainate and metabotropic glutamate receptors. Neuropharmacology 38:495-504.

Lynch GS, Dunwiddie T, Gribkoff V (1977) Heterosynaptic depression: a postsynaptic correlate of long-term potentiation. Nature 266:737-739.

Malenka RC, Nicoll RA (1999) Long-term potentiation-a decade of progress? Science 285:1870-1874.

Manabe T, Wyllie DJ, Perkel DJ, Nicoll RA (1993) Modulation of synaptic transmission and long-term potentiation: effects on paired pulse facilitation and EPSC variance in the CA1 region of the hippocampus. J Neurophysiol 70:1451-1459.

Mulkey RM, Malenka RC (1992) Mechanisms underlying induction of homosynaptic long-term depression in area CA1 of the hippocampus. Neuron 9:967-975.

Nishiyama M, Hong K, Mikoshiba K, Poo MM, Kato K (2000) Calcium stores regulate the polarity and input specificity of synaptic modification. Nature 408:584-588.

Oliet SH, Malenka RC, Nicoll RA (1997) Two distinct forms of long- 
term depression coexist in CA1 hippocampal pyramidal cells. Neuron 18:969-982.

Scanziani M, Malenka RC, Nicoll RA (1996) Role of intercellular interactions in heterosynaptic long-term depression. Nature 380:446-450.

Staubli UV, Ji ZX (1996) The induction of homo- vs. heterosynaptic LTD in area CA1 of hippocampal slices from adult rats. Brain Res 714:169-176.

Stevens CF, Wang Y (1994) Changes in reliability of synaptic function as a mechanism for plasticity. Nature 371:704-707.

Teyler TJ, Cavus I, Coussens C (1995) Synaptic plasticity in the hippocampal slice: functional consequences. J Neurosci Methods 59:11-17.

Thiels E, Barrionuevo G, Berger TW (1994) Excitatory stimulation during postsynaptic inhibition induces long-term depression in hippocampus in vivo. J Neurophysiol 72:3009-3016.
Thomas MJ, Moody TD, Makhinson M, O’Dell TJ (1996) Activitydependent beta-adrenergic modulation of low frequency stimulation induced LTP in the hippocampal CA1 region. Neuron 17:475482.

Wagner JJ, Alger BE (1995) GABAergic and developmental influences on homosynaptic LTD and depotentiation in rat hippocampus. J Neurosci 15:1577-1586.

Wigstrom H, Gustafsson B (1985) Facilitation of hippocampal longlasting potentiation by GABA antagonists. Acta Physiol Scand 125:159-172.

Yang SN, Tang YG, Zucker RS (1999) Selective induction of LTP and LTD by postsynaptic $\left[\mathrm{Ca}^{2+}\right]_{\mathrm{i}}$ elevation. J Neurophysiol 81:781-787.

Zucker RS (1989) Short-term synaptic plasticity. Annu Rev Neurosci 12:13-31. 